\title{
EAl Endorsed Transactions

\section{Analysis of a FTN Multicarrier System: Interference Mitigation Based on Tight Gabor Frames}

\author{
Alexandre Marquet ${ }^{1,2, *}$, Cyrille Siclet ${ }^{1}$, Damien Roque ${ }^{2}$, Pierre Siohan ${ }^{3}$ \\ ${ }^{1}$ Univ. Grenoble Alpes, GIPSA-Lab, F-38400 Grenoble, France. \\ ${ }^{2}$ Institut Supérieur de l'Aéronautique et de l'Espace (ISAE-SUPAERO), Univ. Toulouse, F-31055 Toulouse, France. \\ ${ }^{3}$ (Retired from) Orange-Labs, F-35512 Cesson-Sevigné, France.
}

\section{Abstract}

Cognitive radio applications require flexible waveforms to overcome challenges such as opportunistic spectrum allocation. In this context, filtered multicarrier modulations are particularly justified to fit timefrequency characteristics of the channel.

In our theoretical framework, a multicarrier signal is described as a Gabor family the coefficients of which are the symbols to be transmitted and the generators are the time-frequency shifted pulse shapes to be used. In this article, we consider non-rectangular pulse shapes and an increased signaling density such that inter-pulse interference is unavoidable.

Such an interference is minimized when using a tight Gabor frame. We show that, in this case, it can be approximated as an additive Gaussian noise, allowing us to compute theoretical bit-error-probability. This result allows to predict the convergence of a coded system using iterative decoding. We also study the relevancy of such a system in an interference cancellation context.

Received on 01 June 2016; accepted on 20 January 2017; published on 23 February 2017

Keywords: multicarrier modulations, faster-than-Nyquist signaling, linear system, optimal pulse-shapes, Gabor frames, interference analysis, interference cancellation, low-density parity check codes.

Copyright (C) 2017 Alexandre Marquet et al., licensed to EAI. This is an open access article distributed under the terms of the Creative Commons Attribution license (http://creativecommons.org/licenses/by/3.0/), which permits unlimited use, distribution and reproduction in any medium so long as the original work is properly cited.

doi:10.4108/eai.23-2-2017.152191

\section{Introduction}

In most of current communication systems, the linear part allows for perfect symbol reconstruction: the synthesis and analysis families used in the transmitter and the receiver form biorthogonal frames (also known as Riesz bases). In a single-carrier bandlimited communication scenario, this requires the Nyquist criterion to be respected [1]. In other words, the transmission rate must be lower than the bilateral bandwidth of the transmitted signal.

With an increasing need of spectral efficiency driven by overcrowded frequency bands, the main strategy relies on an increase of constellation size given a constant transmission power, bandwidth and

\footnotetext{
*Corresponding author. Email: alexandre.marquet@gipsalab.grenoble-inp.fr
}

symbol rate (below the Nyquist limit). This choice induces a decrease in the minimum Euclidean distance between transmitted signals, thus becoming more sensitive to noise and consequently increasing bit-errorprobability [2].

A more unusual way to improve spectral efficiency is to increase the symbol rate until the Nyquist criterion is overridden, leading to unavoidable interpulse interference (IPI). This idea has been proposed by J. Mazo under the denomination "faster-thanNyquist" (FTN) [3]. He showed that an increase up to approximately $25 \%$ of the Nyquist symbol rate keeps the minimal Euclidean distance between signals unchanged. As a consequence, considering the work of G.D. Forney on the optimal detection in presence of inter-symbol interference, one can preserve an acceptable bit-error-probability at the price of a greater 
computational complexity at the receiver side [2] (e.g., maximum likelihood approaches).

FTN transmission techniques can be extended to multicarrier modulations [4]. In this case, denoting $F_{0}$ the inter-carrier spacing and $T_{0}$ the multicarrier symbol duration, it can be shown that if the density $\rho=1 /\left(F_{0} T_{0}\right)$ of the system is strictly greater than one, then the synthesis and analysis families, respectively used for transmission and reception, can no longer be biorthogonal but can still form overcomplete frames [5]. This leads to IPI both in time and/or frequency. Numerous studies focus on the realization of coded multicarrier FTN systems using, in particular, serial or parallel concatenations [6] as well as turboequalization techniques [7]. Studies of these latter systems over additive white Gaussian noise (AWGN) channels show great performance, confirming their relevance, even if their intrinsic complexity makes their design and performance comparison particularly demanding in terms of simulation time.

In cognitive radio applications, multicarrier waveforms are widely used due to their flexibility in terms of frequency allocation combined with high spectral efficiency schemes (i.e., overlapping orthogonal pulse shapes) $[8,9]$. Here, the FTN context introduces the density as a new degree of freedom: a trade-off between spectral efficiency and decoding computational complexity can be set according to application's requirements (e.g., energy consumption, throughput...) and channel's conditions (e.g., thermal noise power, timefrequency selectivity, synchronization impairments...). Besides, since FTN systems relax the orthogonality constraint, it enables new optimizations criteria for pulse shapes design such as: frequency localization intended for white spaces opportunistic filling ; peak-to-average power ratio to alleviate power amplification efficiency issues...

In this article, we study a linear multicarrier system operating with overcomplete Gabor frames, as it plays a fundamental role in practical systems, including decision feedback and iterative structures (e.g., turboequalizers). Our work includes guidelines for the design of such systems over an AWGN channel, based on the parameters of the linear part of the system. First of all, we focus on the closed-form expression of the bit-error-probability of our linear system provided that tight frames are used, as prescribed in [10] in order to maximize the signal to interference plus noise ratio (SINR). Secondly, we show how the bit-error-probability closed-form expression of the linear system can be used to guide the design of more complex structures (including iterative receivers such as turboequalizers). Finally, we investigate the behavior of interference cancellation receivers for FTN multicarrier systems.

The structure of this article is as follows. Part 2 details the input-output relations of the system in presence of noise, based on the frame theory. This theoretical framework allows for the determination of the SINR, and the theoretical bit-error-probability, based on the assumption of normality of the interference, that are assessed by means of simulations. Part 3 shows how coded multicarrier FTN systems based on tight frames can be used together with soft-decoding algorithms, and an example of how our closed-form expression of the error probability can predict their performances is presented through simulations using low-density parity check (LDPC) codes. The last part analyzes the relevance of interference cancellation techniques and also validates the relevance of using tight frames in this context. Finally, conclusions and insights are presented in Part 5.

Notation: Uppercase bold letters denote sets (e.g., I) with cardinality $|\mathbf{I}|$. Particularly, let $\mathbf{C}$ be the set of complexes, $\mathbf{R}$ the set of reals and $\mathbf{Z}$ the set of integers. Vector spaces use calligraphic letters (e.g., $\mathcal{H}$ ). Lowercase bold letters denote vector families (e.g., g). Plaintext, either lowercase or uppercase denote functions, vectors (e.g., $f(t))$ or scalars. We use $(\cdot)^{*}$ to denote complex conjugate, $\operatorname{Pr}\{\cdot\}$ probability, $\mathrm{E}(\cdot)$ expectation, $\delta(\cdot)$ Dirac delta function.

Let $x(t), t \in \mathbf{R}$ and $y[k], k \in \mathbf{Z}$ be two complexvalued functions. For each case, we define for $p \in\{1,2\}$ the spaces $\mathcal{L}_{p}(\mathbf{R})=\left\{x: \mathbf{R} \rightarrow \mathbf{C} / \int_{-\infty}^{+\infty}|x(t)|^{p} \mathrm{~d} t<+\infty\right\}$ and $\ell_{p}(\mathbf{I})=\left\{y: \mathbf{I} \rightarrow \mathbf{C} / \sum_{k \in \mathbf{I}}|y[k]|^{p}<+\infty\right\}$, with $\mathbf{I}$ a countable set. The inner product is defined by

$$
\left\langle x_{1}, x_{2}\right\rangle \stackrel{\text { def }}{=} \int_{-\infty}^{+\infty} x_{1}^{*}(t) x_{2}(t) \mathrm{d} t \quad \forall x_{1}(t), x_{2}(t) \in \mathcal{L}_{2}(\mathbf{R})
$$

and the $\mathcal{L}_{2}$-norm is given by $\left\|x_{1}\right\|=\sqrt{\left\langle x_{1}, x_{1}\right\rangle}$.

\section{Optimal linear multicarrier system in presence of white Gaussian noise}

\subsection{Inpułoutputrelation}

Let us denote $\boldsymbol{c}=\left\{c_{m, n}\right\}_{(m, n) \in \boldsymbol{\Lambda}} \in \ell_{2}(\boldsymbol{\Lambda})$ with $\boldsymbol{\Lambda} \subset \mathbf{Z}^{2}$, a sequence of zero-mean, independent and identically distributed (IID) coefficients taking their values in a finite alphabet $\mathbf{A} \subset \mathbf{C}$, with variance $\sigma_{c}^{2}$. The multicarrier signal is then written as:

$$
s(t)=\sum_{(m, n) \in \boldsymbol{\Lambda}} c_{m, n} g_{m, n}(t) \quad \forall t \in \mathbf{R}
$$

with $g=\left\{g_{m, n}\right\}_{(m, n) \in \boldsymbol{\Lambda}}$ a Gabor family, with parameters $F_{0}, T_{0}>0$ and the elements of which are given by the generator $g(t) \in \mathcal{L}_{2}(\mathbf{R})$ such that:

$$
g_{m, n}(t)=g\left(t-n T_{0}\right) e^{j 2 \pi m F_{0} t} .
$$

As a result, the information carried by $c$ is regularly spread in the time-frequency plane (Fig. 1) with a minimum distance $F_{0}$ in frequency and $T_{0}$ in time. 


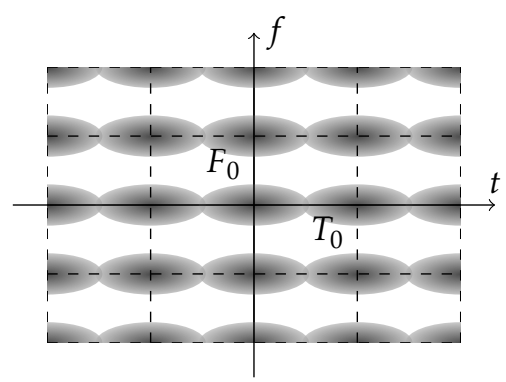

Figure 1. Representationof a transmittedsignal in the timefrequencyplane. Here, the generator $g$ and the parametersof the lattice allow for a separation in the frequencydomainbut not in the timedomain.

In a real case scenario, we usually have $\Lambda=$ $\{0, \ldots, M-1\} \times\{0, \ldots, K-1\}$ where $M, K$ are strictly positive integers representing respectively the number of subcarriers and the number of multicarrier symbols to be transmitted. Such a restriction to a finite signaling set induces the convergence of the sum in (2). Nevertheless it can still contain a large amount of terms, so it is important to make it stable. Denoting $\mathcal{H}_{g}=$ $\overline{\operatorname{Vect}}(\boldsymbol{g})$ the closure of the linear span of the family $\boldsymbol{g}^{1}$, the stability of (2) is guaranteed when $g$ is a Bessel sequence, which means that we can find an upper bound $B_{g}>0$ such as:

$$
\sum_{(m, n) \in \boldsymbol{\Lambda}}\left|\left\langle g_{m, n}, x\right\rangle\right|^{2} \leq B_{g}\|x\|^{2} \quad \forall x \in \mathcal{H}_{g} .
$$

In order to retrieve the data $c$ from the knowledge of $s(t)$, it is furthermore necessary (and sufficient) for $g$ to be a linearly independent family. Hence $g$ should be a Riesz basis of $\mathcal{H}_{g}$, in other words a linearly independent family for which we can find $0<A_{g} \leq B_{g}$ such that:

$$
A_{g}\|x\|^{2} \leq \sum_{(m, n) \in \Lambda}\left|\left\langle g_{m, n}, x\right\rangle\right|^{2} \leq B_{g}\|x\|^{2} \quad \forall x \in \mathcal{H}_{g}
$$

In this case, the density $\rho$ of $g$ is necessarily lower than or equal to one [11]. On the contrary, in order to improve the spectral efficiency of the system (for a fixed number of bits per symbol), this article focuses on the case where $\rho>1$. However, this increase in spectral efficiency is counterbalanced by an induced interference. In a linear receiver, this interference can be considered as a noise leading to an increased error probability. Indeed, when $\rho>1$, $g$ is necessarily a linearly dependent Gabor family. Yet, it can also be an overcomplete frame of $\mathcal{L}_{2}(\mathbf{R})$,

\footnotetext{
${ }^{1}$ The closure of a normed vector space $\mathbf{E}$ contains all the elements of E, together with its limit elements. For example, the closure of the set of the rational numbers is the set of the real numbers.
}

i.e., a linearly dependent family for which (5) is valid not only for $x \in \mathcal{H}_{g}$, but for every $x \in \mathcal{L}_{2}(\mathbf{R})$, which guarantees that (2) is always stable and $\mathcal{H}_{g}=\mathcal{L}_{2}(\mathbf{R})$. However, $g$ cannot be a basis of $\mathcal{L}_{2}(\mathbf{R})$. Thus, it is possible that $\sum_{(m, n) \in \Lambda} c_{m, n} g_{m, n}(t)=\sum_{(m, n) \in \Lambda} c_{m, n}^{\prime} g_{m, n}(t)$ with $c_{m, n} \neq c_{m, n}^{\prime}$. But there exist dual frames $\check{g}$ with bounds $A_{\check{g}}$ and $B_{\check{g}}$ such that for all $s(t) \in \mathcal{L}_{2}(\mathbf{R}), s(t)=$ $\sum_{(m, n) \in \Lambda}\left\langle\check{g}_{m, n}, s\right\rangle g_{m, n}(t)=\sum_{(m, n) \in \Lambda}\left\langle g_{m, n}, s\right\rangle \check{g}_{m, n}(t)$. Among all of the dual frames, one is called the dual canonical dual frame and is such that $A_{\check{g}}=1 / B_{g}$ and $B_{\check{g}}=1 / A_{g}$. What is more, a frame $g$ is said to be tight when $A_{g}=B_{g}$. And, in this case, the canonical dual frame is $\check{g}=1 / A_{g} g$ so that $s(t)=\frac{1}{A_{g}} \sum_{(m, n) \in \boldsymbol{\Lambda}}\left\langle g_{m, n}, s\right\rangle g_{m, n}(t)$ for all $s(t) \in \mathcal{L}_{2}(\mathbf{R})$ if $\boldsymbol{g}$ is a tight frame.

A linear receiver is considered as a first stage of a more complete FTN system (necessarily non-linear in order to yield acceptable performance). In this context, the symbols $\hat{c}=\left\{\hat{c}_{p, q}\right\}_{(p, q) \in \Lambda}$ estimated by this linear stage are given by:

$$
\hat{c}_{p, q}=\left\langle\check{g}_{p, q}, r\right\rangle \quad \forall(p, q) \in \Lambda
$$

where $\check{g}=\left\{\check{g}_{m, n}\right\}_{(m, n) \in \Lambda}$ is a reception family, $r(t)=s(t)+$ $n(t)$ is the signal seen by the receiver and $n(t)$ is a zero-mean white Gaussian complex circular noise independent from the symbols, with bilateral power spectral density $\gamma_{n}(f)=2 N_{0}$ for $f \in \mathbf{R}: \mathrm{E}(n(t))=0$ and $\mathrm{E}\left(n^{*}(t) n\left(t^{\prime}\right)\right)=2 N_{0} \delta\left(t-t^{\prime}\right)$.

\subsection{Interferenceand noise analysis}

By rewriting (6), we can clearly identify the interference and noise terms:

$$
\begin{aligned}
\hat{c}_{p, q}=\underbrace{c_{p, q}\left\langle\check{g}_{p, q}, g_{p, q}\right\rangle}_{\tilde{c}_{p, q} \text { : useful signal }} & \\
+\underbrace{\sum_{(m, n) \in \Lambda \backslash\{(p, q)\}} c_{m, n}\left\langle\check{g}_{p, q}, g_{m, n}\right\rangle}_{i_{p, q} \text { :interference }} & +\underbrace{\left\langle\check{g}_{p, q}, n\right\rangle}_{n_{p, q} \text { :noise }} .
\end{aligned}
$$

We already showed in [10] that the signal to interference ratio (SIR) is maximized when $\check{g}$ and $g$ are dual canonical Gabor frames, and the SINR is maximized when $\boldsymbol{g}$ is a tight Gabor frame and $\check{\boldsymbol{g}}=1 / A_{g} \boldsymbol{g}$. This leads 
to the following expressions:

$$
\begin{aligned}
E_{s} & =\frac{1}{2} \sigma_{c}^{2}\|g\|^{2}=\frac{\sigma_{c}^{2} A_{g}}{2 \rho}, \\
\sigma_{i}^{2} & =\mathrm{E}\left(\left|i_{p, q}\right|^{2}\right)=(\rho-1) \sigma_{c}^{2}, \\
\sigma_{n}^{2} & =\mathrm{E}\left(\left|n_{p, q}\right|^{2}\right)=2 \frac{\rho}{A_{g}} N_{0},
\end{aligned}
$$

with $E_{s}$ the per-symbol energy, $\sigma_{i}^{2}$ the variance of the interference and $\sigma_{n}^{2}$ the variance of the filtered noise. The SINR is then written as

$$
\operatorname{SINR}=\frac{1}{\rho-1+\frac{N_{0}}{E_{s}}} .
$$

We can see from (7) that the interference term $i_{p, q}$ is a random variable independent from the noise and corresponding to the sum of a large number of random variables $\tilde{c}_{m, n}$ which are zero-mean, independent, following the same kind of law but with different variances $\sigma_{\tilde{c}_{m, n}}^{2}$ :

$$
\tilde{c}_{m, n}=c_{m, n}\left\langle\check{g}_{p, q}, g_{m, n}\right\rangle,
$$

and

$$
\sigma_{\tilde{c}_{m, n}}^{2}=\sigma_{c}^{2}\left|\left\langle\check{g}, g_{m-p, n-q}\right\rangle\right|^{2} .
$$

All the conditions for applying the central limit theorem are thus not fulfilled but, as shown by our simulations in Part 2.3, the Gaussian approximation is accurate for the sake of error-probability estimation. That is why in the following, we will assume the interference $i_{p, q}$ to be a normal zero-mean complex circular random variable independent from the noise. This is analogous to a case where the symbols would have been transmitted through an AWGN channel characterized by a signal-to-noise ratio given by (11). It is interesting to note that the noise term $n_{p, q}$ is zeromean and Gaussian, but not necessarily white.

Simulations. The simulations presented in this part consist in the transmission of $K=5000$ multicarrier symbols over $M=128$ subcarriers with a QPSK constellation. They were run for various generators generating tight frames or not. Tight frames are obtained using the duality principle of Wexler-Raz theorem [5, theorem 9.3.4]. Indeed, this theorem states that $g$ and $\check{g}$ generate dual Gabor frames with time-frequency parameters $T_{0}, F_{0}$ if and only if they generate biorthogonal Riesz Gabor sequences with time-frequency parameters $1 / F_{0}, 1 / T_{0}$. What is more, $g$ generates a tight Gabor frame with timefrequency parameters $T_{0}, F_{0}$ if and only if it generates an orthogonal Gabor sequence with parameters $1 / F_{0}$, $1 / T_{0}$. Thus, orthogonal generators used in the case $\rho<$ 1 correspond to tight frame generators when $\rho>1$.
Table 1. Proprieties of the generators used in our simulations.

\begin{tabular}{c|c|c|c}
$\begin{array}{c}\text { Transmission } \\
\text { generator } g\end{array}$ & $\begin{array}{c}\text { Reception } \\
\text { generator } \check{g}\end{array}$ & $\begin{array}{c}\text { Canonical } \\
\text { dual frames }\end{array}$ & $\begin{array}{c}\text { Tight frame with } \\
\|g\|=1 / \sqrt{\rho}\end{array}$ \\
\hline \hline TFL & $\check{g}=g$ & Yes & Yes \\
\hline OBE & $\check{g}=g$ & Yes & Yes \\
\hline $\begin{array}{c}\check{\text { SRRC }}(\alpha=\rho-1) \\
(\check{g}=g\end{array}$ & Yes & Yes \\
\hline $\operatorname{RECT}_{T_{0}}$ & $\check{g}=g$ & Yes & Yes \\
\hline $\operatorname{RECT}_{\rho T_{0}}$ & RECT $_{T_{0}}$ & No & No \\
\hline $\operatorname{RECT}_{\rho T_{0}}$ & $\check{g}=g$ & No & No \\
\hline
\end{tabular}

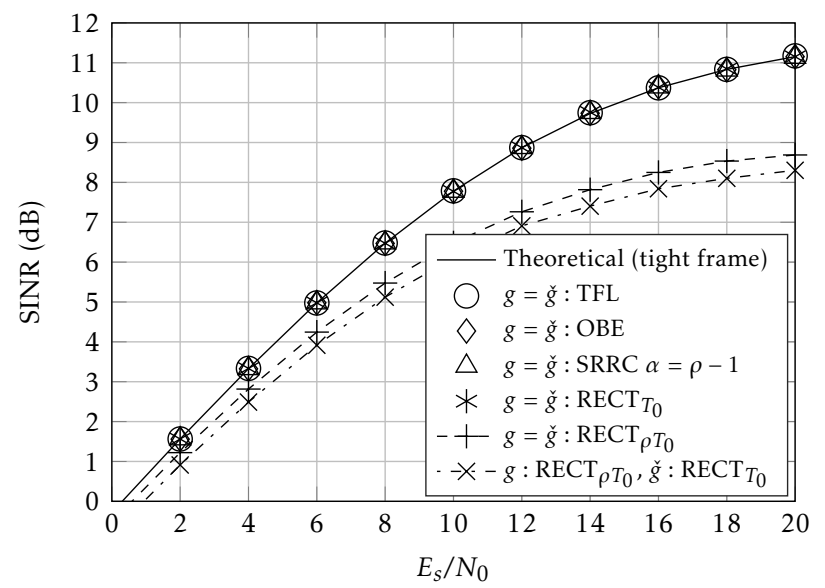

Figure 2. SINR as a functionof $E_{S} / N_{0}$, with $\rho=16 / 15$.

Thus, the orthogonal generators obtained [12] form tight frames, as shown in [10]. The interest of these generators is that they have minimal length which reduces the complexity of the system and also allows that interference is spread over few symbols. Moreover, they maximize the time-frequency localization (TFL) or minimize the out-of-band energy (OBE). For the same reasons, the square-root-raised-cosine (SRRC) with rolloff factor $\alpha=\rho-1$ as well as the $T_{0}$-width rectangular $\left(\mathrm{RECT}_{T_{0}}\right)$ generator yield tight frames. When such a generator is used both in transmission and reception, it is sufficient to set its norm to $1 / \sqrt{\rho}$ in order to obtain tight frames with $A_{g}=1$. By contrast, although the $\operatorname{RECT}_{\rho T_{0}}$ and $\mathrm{RECT}_{T_{0}}$ generates dual frames, they are not canonical dual so that using one of them for transmission and the other for reception does not lead to a pair of tight frames. Finally, the rectangular generator of width $\rho T_{0}\left(\operatorname{RECT}_{\rho T_{0}}\right)$ does not form canonical dual frames when used both in transmission and reception. This is summarized in Table 1 .

Figure 2 exhibits the perfect prediction of the SINR by (11) when the generators used in transmission and reception form tight frames.

\subsection{Error probabilityapproximation}

We now restrict our analysis to the case where the symbols $c$ are taken from a quadrature phase-shift 
keying (QPSK) constellation. In that case, given the fact that both the noise and the interference are considered Gaussian, the bit error probability for a transmission through an AWGN channel is given by

$$
P_{e}=Q(\sqrt{\mathrm{SINR}})=Q\left(\sqrt{\frac{1}{(\rho-1)+\frac{N_{0}}{2 E_{b}}}}\right)
$$

where $Q(\cdot)$ is the complementary cumulative distribution function (CCDF) of a standard normal distribution and $E_{b}=E_{s} / 2$ the per-bit energy.

Simulations. In this part, we discuss the relevance of the Gaussian approximation of the interference. To this extent, we measure $3.6 \times 10^{6}$ realizations of the interference $i_{p, q}$ term by performing a transmission of $M=64$ subcarriers over $K=50000$ multicarrier symbols for different values of $\rho$, using a QPSK constellation and tight frames. The variance of the obtained samples is then normalized thus giving standardized versions of $i_{p, q}$ depending on $\rho$ with comparable empirical probability density functions and cumulative distribution functions (CDF). The behavior described here has been observed to be similar with both the real and the imaginary part of $i_{p, q}$, and for various generators forming tight frames.

Considering a transmission over a noise-free perfect channel (SINR $=1 /(\rho-1))$, zero-mean IID bits, and denoting $F_{i, \rho}(\cdot)$ the CDF of the interference given a density of $\rho$, we can express the bit-error-probability as:

$$
P_{e}(\rho)=1-F_{i, \rho}(\sqrt{\mathrm{SINR}})=1-F_{i, \rho}\left(\sqrt{\frac{1}{\rho-1}}\right) .
$$

In order to assess the Gaussian approximation, we compare the values of the functions $P_{e}(\rho)$ and $Q(\sqrt{1 /(\rho-1)})$ for various $\rho$ on Figure 3. Even though the interference cannot be characterized by a Gaussian distribution, we can see that the relative approximation error is negligible, except for $\rho$ close to one, in this context of error probability estimation. Our simulations furthermore revealed that the Gaussian approximation then constitutes an upper bound for the bit-errorprobability. This result ensures that the Gaussian approximation can be safely used for multicarrier FTN communication system design and engineering, provided that tight frames are used.

In terms of performance, for this kind of non-coded multicarrier FTN system, Figure 4 shows that the biterror-rate (BER) rapidly rises with the density. We can also see on Figure 5 that a lower-bound of the BER appears when the power of the noise becomes negligible compared to the one of the interference. The limits of the Gaussian approximation of the interference is also highlighted by both figures at strong values of $E_{b} / N_{0}$ and when $\rho$ is close to one (see when $E_{b} / N_{0} \geq 14 \mathrm{~dB}$

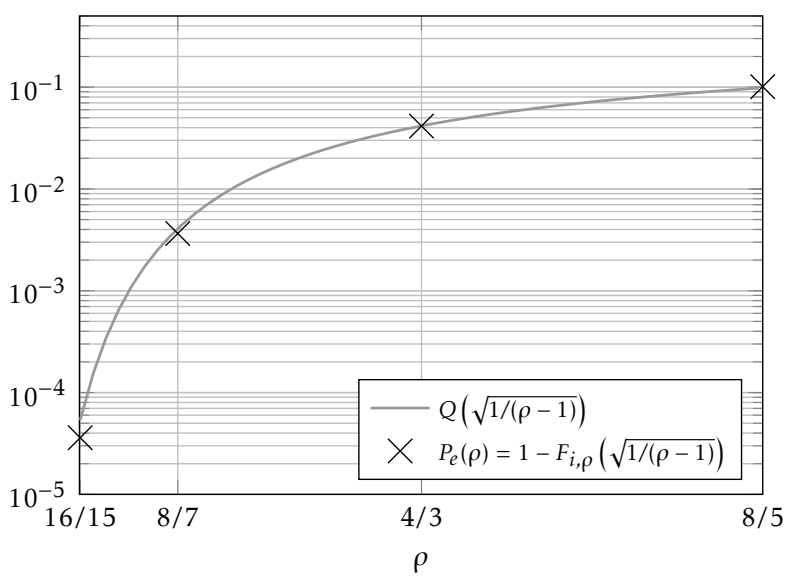

Figure 3. Comparisonof the real CCDF of the interference varyingon $\rho$ and its Gaussian approximation.

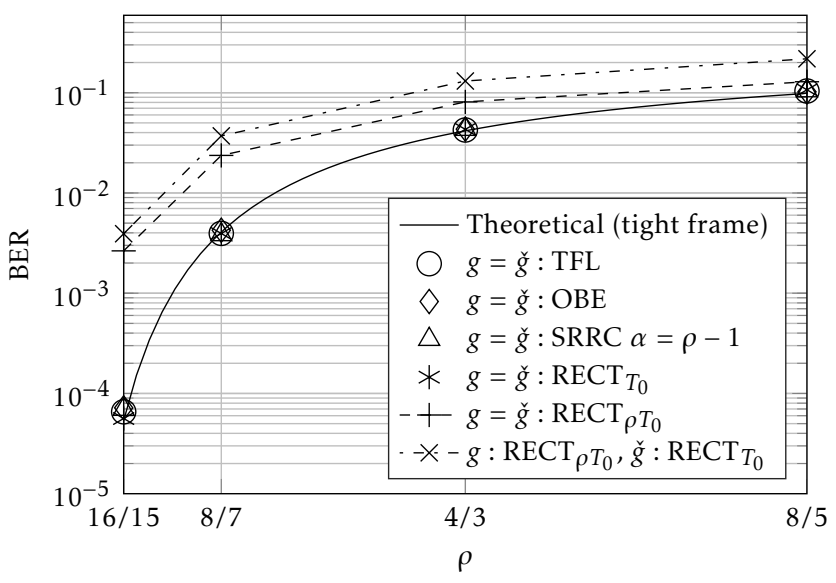

Figure 4. BER as a functionof $\rho$, with $E_{b} / N_{0}=20 \mathrm{~dB}$.

and $\rho=16 / 15)$. In addition, and in accordance with the expression of the SINR, the performance gets worse if the frames used are not tight nor canonical dual. Theses results confirm the needs to develop non-linear detectors allowing for a more efficient IPI mitigation.

\section{Use in a system with iterative decoding}

\subsection{Hypothesisfor log-likelihoodratio computation}

In this section, we take advantage of a LDPC code to mitigate both interference and noise. For optimal performance, decoding algorithm for these codes work with soft inputs in the form of log-likelihood ratios (LLR):

$$
L\left(b_{l}\left(c_{p, q}\right) \mid \hat{c}_{p, q}=\hat{c}\right)=\ln \frac{\operatorname{Pr}\left\{b_{l}\left(c_{p, q}\right)=0 \mid \hat{c}_{p, q}=\hat{c}\right\}}{\operatorname{Pr}\left\{b_{l}\left(c_{p, q}\right)=1 \mid \hat{c}_{p, q}=\hat{c}\right\}},
$$

where $b_{l}(c)$ is the $l$ th bit of the symbol $c$, and $\hat{c} \in \mathbf{C}$ is an observation of the random variable $\hat{c}_{p, q}$. Given that events $c_{p, q}=c$ and $c_{p, q}=c^{\prime}\left(c \neq c^{\prime}\right)$ are mutually 


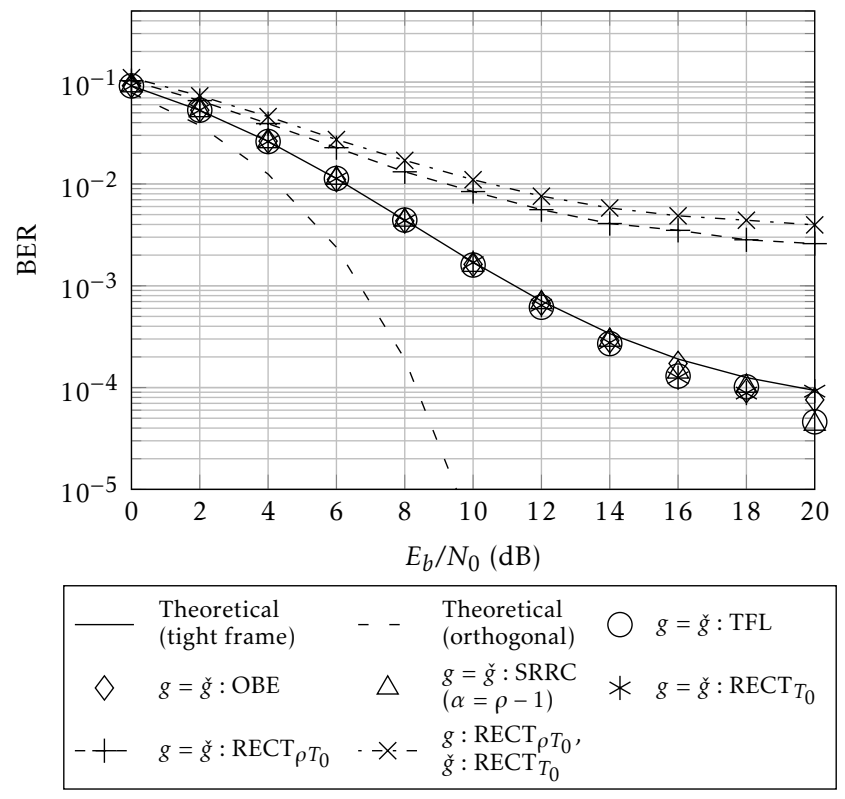

Figure 5. BER as a functionof $E_{b} / N_{0}$, with $\rho=16 / 15$.

exclusive, probability for the $l$ th bit of a symbol $c_{p, q}$ to take one particular value $b \in\{0,1\}$ can be obtained as:

$$
\operatorname{Pr}\left\{b_{l}\left(c_{p, q}\right)=b \mid \hat{c}_{p, q}=\hat{c}\right\}=\sum_{c \in \mathbf{A}, b_{l}(c)=b} \operatorname{Pr}_{\mathrm{r}}\left\{c_{p, q}=c \mid \hat{c}_{p, q}=\hat{c}\right\} .
$$

The probability in the sum can be further decomposed, introducing the probability density function (PDF) $f_{\hat{c}_{p, q}}(\cdot)$ of $\hat{c}_{p, q}$, the conditional PDF $f_{\hat{c}_{p, q} \mid c_{p, q}=c}(\cdot)$ of $\hat{c}_{p, q}$ given $c_{p, q}=c$, and thanks to Bayes's theorem:

$$
\operatorname{Pr}\left\{c_{p, q}=c \mid \hat{c}_{p, q}=\hat{c}\right\}=\frac{\operatorname{Pr}\left\{c_{p, q}=c\right\} f_{\hat{c}_{p, q} \mid c_{p, q}=c}(\hat{c})}{f_{\hat{c}, q}(\hat{c})} .
$$

Here, combining (17) and (18) in (16) and using the hypothesis that the transmitted symbols $c_{p, q}$ are IID with uniform distribution, so that $\operatorname{Pr}\left\{c_{p, q}=c\right\}=1 /|\mathbf{A}|$, we obtain:

$$
L\left(b_{l}\left(c_{p, q}\right) \mid \hat{c}_{p, q}=\hat{c}\right)=\ln \frac{\sum_{c \in \mathbf{A}, b_{l}(c)=0} f_{\hat{c}_{p, q} \mid c_{p, q}=c}(\hat{c})}{\sum_{c \in \mathbf{A}, b_{l}(c)=1} f_{\hat{c}_{p, q} \mid c_{p, q}=c}(\hat{c})} .
$$

In order to compute the PDF in the sums of (19), we define the random variable $v_{p, q}=n_{p, q}+i_{p, q} \in \mathbf{C}$. Let us consider the noise and the interference as two zeromean, independent complex circular Gaussian random variables: $n_{p, q} \sim \mathcal{C N}\left(0, \sigma_{n}^{2}\right)$ and $i_{p, q} \sim \mathcal{C N}\left(0, \sigma_{i}^{2}\right)$. Then $v_{p, q}$ is also a complex circular random variable: $v_{p, q} \sim$ $\mathcal{C N}\left(0, \sigma_{v}^{2}\right)$, with $\sigma_{v}^{2}=\sigma_{n}^{2}+\sigma_{i}^{2}$. Furthermore, from (7), we can write $\hat{c}_{p, q}=c_{p, q}\left\langle\check{g}_{p, q}, g_{p, q}\right\rangle+v_{p, q}$, so that the law of $\hat{c}_{p, q}$ conditionally to $c_{p, q}=c$ is a linear transformation of the Gaussian random variable $v_{p, q}$. Its PDF is then written as follows:

$$
f_{\hat{c}_{p, q} \mid c_{p, q}=c}(\hat{c})=\frac{1}{\pi \sigma_{v}^{2}} \exp \frac{-\left|\hat{c}-c\left\langle\check{g}_{p, q}, g_{p, q}\right\rangle\right|^{2}}{\sigma_{v}^{2}} .
$$

Considering transmission and reception generators leading to tight frames, we can exploit (8), (9) and (10):

$$
\begin{gathered}
\sigma_{v}^{2}=(\rho-1) \sigma_{c}^{2}+\frac{\rho}{A_{g}} 2 N_{0} \\
\left\langle\check{g}_{p, q}, g_{p, q}\right\rangle=\frac{1}{\rho} .
\end{gathered}
$$

As a conclusion, combining (22) and (20) into (19) gives the following expression of the LLR:

$$
L\left(b_{l}\left(c_{p, q}\right) \mid \hat{c}_{p, q}=\hat{c}\right)=\ln \frac{\sum_{c \in \mathbf{A}, b_{l}(c)=0} \exp \frac{-|\hat{c}-c / \rho|^{2}}{\sigma_{v}^{2}}}{\sum_{c \in \mathbf{A}, b_{l}(c)=1} \exp \frac{-|\hat{c}-c / \rho|^{2}}{\sigma_{v}^{2}}},
$$

under these constraints:

1. the input symbols are IID with uniform distribution;

2. interference and noise are independent with zeromean complex circular Gaussian distribution;

3. transmission and reception generators form a tight frame.

\subsection{Semi-analytical performancessessmentmethod}

BER curves of systems using powerful coding schemes such as turbocodes or LDPC are characterized by a socalled "convergence threshold" [13] which is the $E_{b} / N_{0}$ value from which the coded system achieves better performance than the uncoded one. Given an AWGN channel, it is also possible to characterize the coded system by a curve presenting the BER at the output of the decoder (denoted as "output BER" - BER out) varying with the BER at the input of the decoder (denoted as "input BER" - BER in ) as in Figure 6. On this kind of curve, the convergence threshold is found at a given input BER. As a consequence, and thanks to the expression of the bit-error probability (14), it is possible to find the optimal density $\rho$ allowing the coded system to converge given an arbitrary value of $E_{b} / N_{0}$.

Simulations. As an example, Figure 6 shows that a coded system using the LDPC code of rate $1 / 2$ specified in the DVB-S2 specification [14] has its convergence threshold for an input BER of approximately 0.15 . On Figure 7, we can see that when used with a multicarrier FTN system using tight frames, the coded system converges as expected when the input BER goes below 0.15 , at $E_{b} / N_{0}=2 \mathrm{~dB}$. 


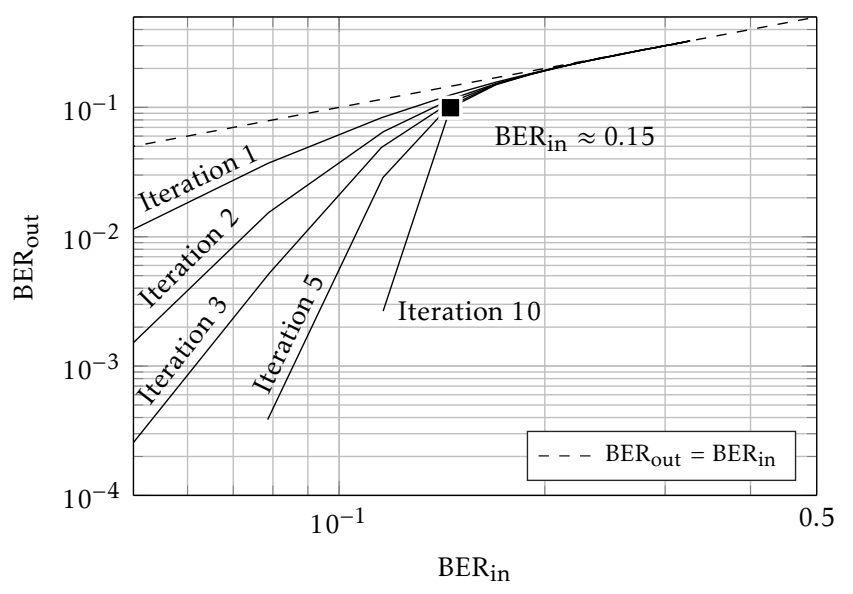

Figure 6. OutputBER as a functionof the input BER for a rate $=1 / 2$ LDPC system. In this configuation, the convergence thresholdis at an inputBER of 0.15 .

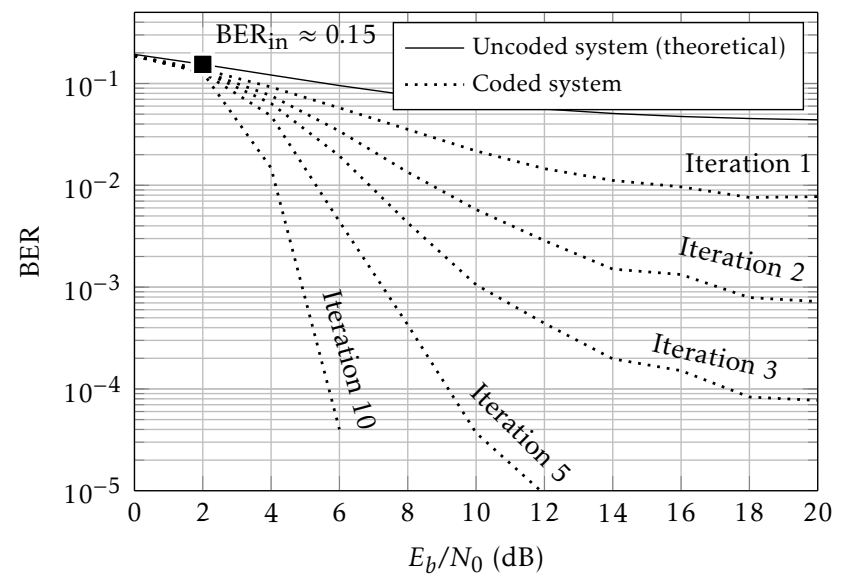

Figure 7. BER as a functionof $E_{b} / N_{0}$ using a rate $1 / 2 \mathrm{LDPC}$ code, $\rho=4 / 3$ and a TFL generator for 10 iterations of the decoder

\section{Interference cancellation based on a pseudo-genie receiver}

From the expression of the bit error probability (14), as expected, the FTN linear system shows worse performance compared to the orthogonal case. Besides, from the expression of the received signal (7), one can notice that the performance of the orthogonal system can be retrieved by removing the interference induced by the FTN system, enabling a spectral efficiency improvement while keeping the BER unchanged.

Such an interference cancellation (IC) is usually performed by estimating the received symbols, then computing the interference term from those estimates and subtracting it to the received signal. Consequently, one should keep in mind that symbols at the IC's output depend on those at the output of the linear receiver [15].
Given that this estimation might not be perfect, it is interesting to assess the behavior of this system in presence of errors on the estimated symbols. To fulfill that purpose without having to restrict ourselves to a particular method for computing the estimated symbols, we implemented the pseudo-genie receiver depicted by Figure 8 . The difference with a "true genie" receiver is that its knowledge of the transmitted symbols is corrupted by a binary symmetric channel inducing errors on the bits used to compute the interference term. Thus, the symbols $\left\{\bar{c}_{p, q}\right\}_{(p, q) \in \Lambda}$ at the output of the pseudo-genie receiver are given by

$$
\begin{aligned}
\bar{c}_{p, q}=c_{p, q}\left\langle\check{g}_{p, q}, g_{p, q}\right\rangle & \\
+\sum_{(m, n) \in \Lambda \backslash\{(p, q)\}}\left(c_{m, n}-c_{m, n}^{\prime}\right)\left\langle\check{g}_{p, q}, g_{m, n}\right\rangle & +\left\langle\check{g}_{p, q}, n\right\rangle,
\end{aligned}
$$

where each bit of each element of $\left\{c_{m, n}^{\prime}\right\}_{(m, n) \in \Lambda}$ are corrupted by a binary symmetric channel:

$$
\begin{aligned}
& \forall(m, n) \in \Lambda, l \in\{0, \ldots,|\mathbf{A}|-1\} \\
& \begin{cases}\operatorname{Pr}_{\mathrm{r}}\left\{b_{l}\left(c_{m, n}^{\prime}\right) \neq b_{l}\left(c_{m, n}\right)\right\} & =P_{e, \text { genie }} \\
\operatorname{P}_{\mathrm{r}}\left\{b_{l}\left(c_{m, n}^{\prime}\right)=b_{l}\left(c_{m, n}\right)\right\} & =1-P_{e, \text { genie }}\end{cases}
\end{aligned}
$$

From (24), we consider two cases.

- If $P_{e \text {,genie }}=0$ (which implies $c_{m, n}^{\prime}=c_{m, n}$ ), the whole interference is canceled. In this case, ensuring $\check{\boldsymbol{g}}=\alpha \boldsymbol{g}(\alpha>0)$ is sufficient for the linear part of the receiver to act as a matched filter (such that the SNR is maximized), ensuring the same performance for this equalized system and the orthogonal system.

- If $P_{e, \text { genie }}>0$, it is valuable to use tight frames, as they also maximize the SINR obtained after interference cancellation, providing a lower error probability.

Simulations. The performance of this system, simulated by the transmission of $K=5000$ multicarrier symbols over $M=32$ subcarriers using a QPSK constellation. We can see on Figure 9 that it is quite robust to the presence of errors on the bits used to compute and cancel the interference, which gives an insight on how non-linear receivers using interference cancellation (such as decision feedback or turboequalization) could efficiently prevent IPI. Figure 10 shows that generators yielding matched filters and tight frames (respectively $\operatorname{RECT}_{\rho T_{0}}$ and TFL in this simulation) can both reach the error probability of orthogonal systems, provided that few errors are made on the bits used to compute interference. When other generators are used, this lower bound cannot be attained using this interference 


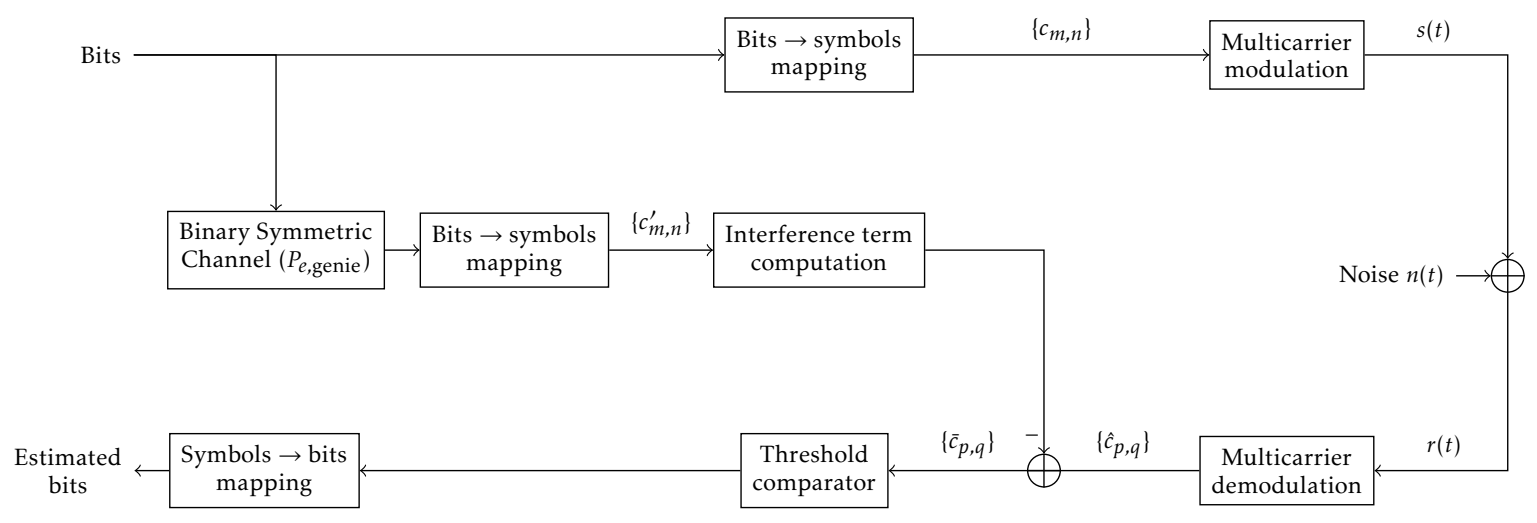

Figure 8. Flowchat of the pseudo-genieinterferencecancellationsystem.

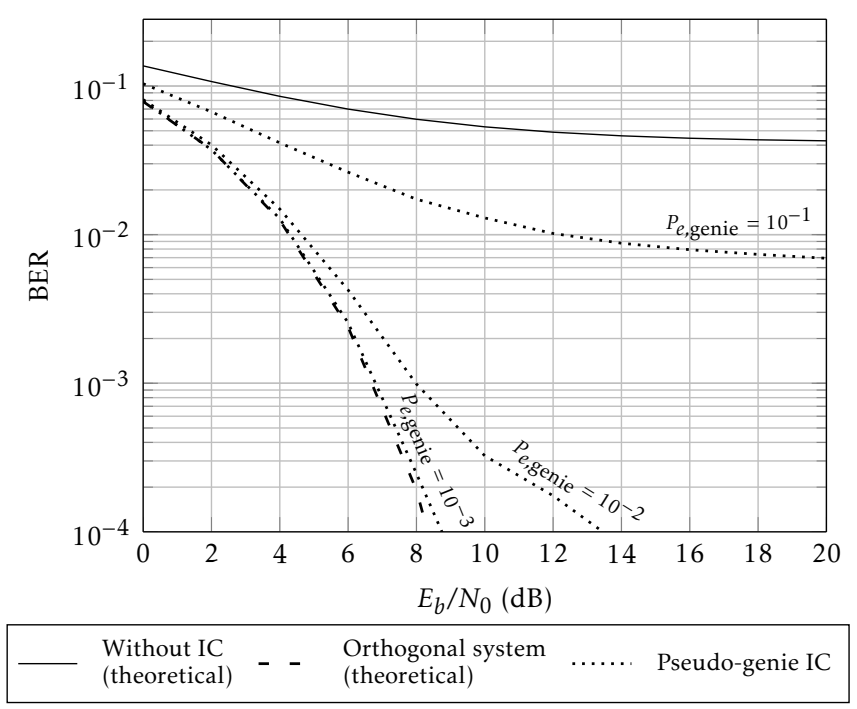

Figure 9. BER as a function of $E_{b} / N_{0}$, with a pseudogenie receiver $\rho=4 / 3$, a TFL generator and $P_{e \text {,genie }} \in$ $\left\{10^{-1}, 10^{-2}, 10^{-3}\right\}$.

cancellation scheme. One can notice that tight frames exhibits better performances over matched filters as the bits used to compute interference get more corrupted (here, when $P_{e \text {,genie }}>10^{-3}$ ). In particular, they provide the smallest region where the BER after interference cancellation is worse than the BER before.

\section{Conclusion}

Through this article, we specified a linear multicarrier system based on the use of overcomplete Gabor frames, enabling an increase in signaling density in the time and/or the frequency domain and leading to a bidimensional FTN system. Consequently, an increase of the spectral efficiency beyond (bi)-orthogonal systems (for a given constellation size) yields interference between pulse-shapes. Such interference can be mitigated by the use of tight frames within the context of a linear system. Furthermore, we showed that interference cancellation

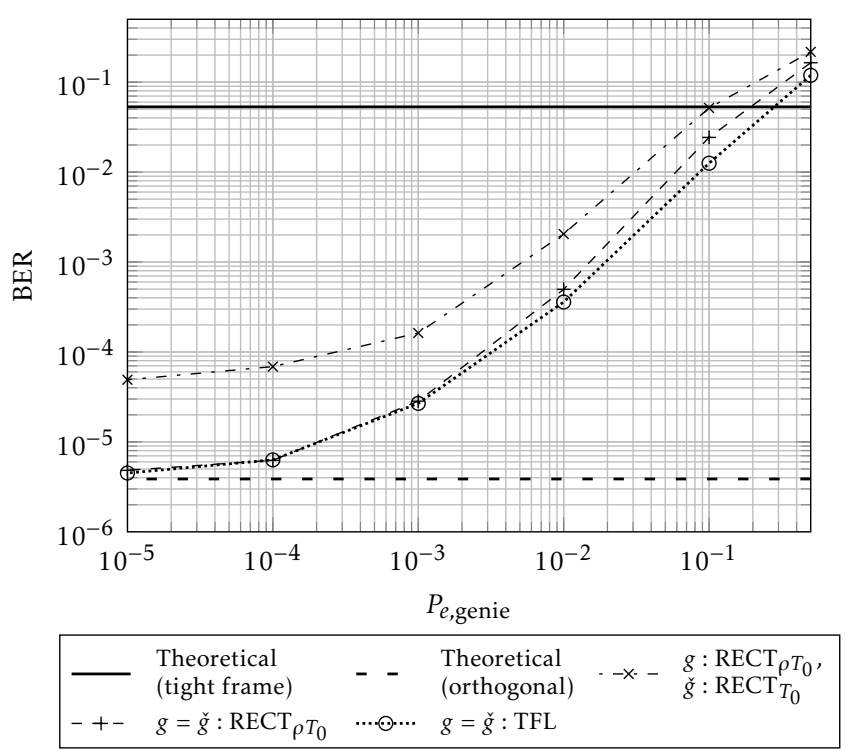

Figure 10. BER as a functionof $P_{e, \text { genie, with a pseudo-genie }}$ receiver $\rho=4 / 3$ and $E_{b} / N_{0}=10 \mathrm{~dB}$.

based on noisy estimates of the transmitted symbols (pseudo-genie receiver) can lead to the same BER as orthogonal systems, but at a higher bitrate.

The results presented in this article allow the ability to relatively compare the performance of FTN multicarrier systems based on the parameters of their linear part (e.g., time-frequency lattice density, transmission/reception filters...). Secondly, we showed how the knowledge of this performance can help the design of more complex receiver structures (e.g., LDPC/turbodecoders, turboequalizers) by predicting their behavior.

Future work may consist in the analysis and efficient implementations of various multicarrier non-linear systems based on tight frames, and transmissions over more complex channels encountered in practical cognitive radio scenarios. 
Acknowledgements. The authors would like to address a particular thank to Dr. Laurent Ros for his valuable advices and relevant remarks concerning this work.

\section{References}

[1] Nyquist, H. (1928) Certain topics in telegraph transmission theory. American Institute of Electrical Engineers, Transactions of the 47(2): 617-644. doi:10.1109/TAIEE.1928.5055024.

[2] Forney, G. (1972) Maximum-likelihood sequence estimation of digital sequences in the presence of intersymbol interference. Information Theory, IEEE Transactions on 18(3): 363-378.

[3] Mazo, J.E. (1975) Faster than Nyquist signaling. Bell System Technical Journal 54: 1451-1462.

[4] Rusek, F. and Anderson, J. (2005) The two dimensional Mazo limit. In Information Theory, 2005. ISIT 2005. Proceedings. International Symposium on: $970-974$. doi:10.1109/ISIT.2005.1523482.

[5] Christensen, O. (2008) Frames and bases: An introductory course (Birkhauser).

[6] Ruser, F. and Anderson, J. (2006) Serial and parallel concatenations based on faster than Nyquist signaling. In Information Theory, 2006 IEEE International Symposium on: 1993 -1997. doi:10.1109/ISIT.2006.261898.

[7] Dasalukunte, D., Rusek, F. and Owall, V. (2011) Multicarrier faster-than-Nyquist transceivers: Hardware architecture and performance analysis. Circuits and Systems I: Regular Papers, IEEE Transactions on 58(4): 827 -838. doi:10.1109/TCSI.2010.2089549.

[8] Mahmoud, H.A., YuceK, T. and Arslan, H. (2009) Ofdm for cognitive radio: merits and challenges. IEEE Wireless Communications 16(2): 615. doi:10.1109/MWC.2009.4907554.

[9] Lin, M., LI, Y., XIAo, L., WANG, J. and Xu, X. (2015) An improved multicarrier based waveform design for cognitive radio communication. In 2015 IEEE 14th International Conference on Cognitive Informatics Cognitive Computing (ICCI ${ }^{*} C$ ) : 122-127. doi:10.1109/ICCICC.2015.7259375.

[10] Siclet, C., Roque, D., Shu, H. and Siohan, P. (2014) On the study of faster-than-Nyquist multicarrier signaling based on frame theory. In Wireless Communications Systems (ISWCS), 2014 11th International Symposium on: 251-255. doi:10.1109/ISWCS.2014.6933356.

[11] Daubechies, I. (1990) The wavelet transform, timefrequency localization and signal analysis. Information Theory, IEEE Transactions on 36(5): 961-1005. doi:10.1109/18.57199.

[12] Pinchon, D. and Siohan, P. (2011) Closed-form expressions of optimal short PR FMT prototype filters. In Proc. IEEE Global Telecommunications Conf. GLOBECOM '11. doi:10.1109/GLOCOM.2011.6133592.

[13] Berrou, C., Amis Cavalec, K., Arzel, M., Glavieux, A., Jezequel, M., Langlais, C., Le Bidan, R. et al. (2007) Codes et turbocodes (sous la direction de Claude Berrou), Iris (Paris: Springer).

[14] (2009), ETSI: EN 302307 Digital Video Broadcasting (DVB); Second generation framing structure, channel coding and modulation systems for Broadcasting, Interactive services, News Gathering and other broadband satellite applications (DVB-S2).

[15] Mueller, M.S. and SAlz, J. (1981) A unified theory of data-aided equalization. The Bell System Technical Journal 60(9): 2023-2038. doi:10.1002/j.15387305.1981.tb00309.x. 